\title{
Downregulation of klotho $\beta$ is associated with invasive ductal carcinoma progression
}

\author{
PING LI $^{1}$, MENG ZHAO $^{1}$, XIAOLI QI $^{1,2}$, XUEGONG ZHU $^{1}$ and JIE DAI ${ }^{1}$ \\ ${ }^{1}$ Department of Pathology, Capital Medical University; ${ }^{2}$ Department of Pathology, \\ Daxing Hospital, Capital Medical University, Beijing 100069, P.R. China
}

Received December 18, 2015; Accepted May 18, 2017

DOI: 10.3892/ol.2017.7110

\begin{abstract}
Klotho $\beta$ (KLB) is a single-pass transmembrane protein measuring 1,043 amino acids in length that shares $41.2 \%$ homology with klotho $\alpha$ (KLA). KLB is a co-receptor and key regulator of the fibroblast growth factor receptor 4 (FGFR4) pathway. KLB interacts with FGFR4 to induce apoptosis and inhibit the proliferation of hepatoma cells, and KLA has been demonstrated to be a tumor suppressor in human breast cancer; however, little is known regarding the role of KLB in breast cancer. In the present study, through an immunohistochemical analysis of invasive ductal carcinoma tissue arrays, low KLB expression was identified in invasive ductal carcinoma samples compared with paired adjacent non-tumorous breast tissues (82 cases). In invasive ductal carcinoma tissues, KLB expression was negatively associated with pathological grade and lymph node metastasis. In 42 cases of paired microdissected breast specimens, the condition of the KLB gene allele was examined to determine the loss of heterozygosity ( $\mathrm{LOH})$, and selective $\mathrm{LOH}$ was identified at the KLB locus in $57.1 \%$ of primary tumors. These data suggest that KLB may be associated with the progression and metastasis of invasive ductal carcinoma, and therefore have clinical and therapeutic importance.
\end{abstract}

\section{Introduction}

Breast cancer is the most common cancer and the first most common cause of cancer-associated mortality in females worldwide (1). The current treatments for breast cancer include surgery, chemotherapy, radiotherapy and hormone therapy, and a small number of patients currently undergo targeted therapy (2).

Correspondence to: Professor Jie Dai or Professor Xuegong Zhu, Department of Pathology, Capital Medical University, 10 Xitoutiao, You'anmen Wai, Fengtai, Beijing 100069, P.R. China

E-mail: sydaijie@163.com

E-mail: zhuxuegong@hotmail.com

Key words: klotho $\beta$, tissue microarray, invasive ductal carcinoma, microsatellite, loss of heterozygosity
The klotho gene family includes klotho $\alpha$ (KLA) and klotho $\beta$ (KLB). KLA is an aging-suppressor gene that encodes a type I membrane protein that is 1,014 amino acids in length (3). The human KLA locus has been assigned to 13 q12 (3-5), and has been demonstrated to be a tumor suppressor in human breast cancer (6). KLB is a single-pass transmembrane protein of 1,043 amino acids in length that is located on 4p14; it shares $41.2 \%$ homology with KLA. KLB is predominantly expressed in the liver, adipose tissue and pancreas (7), and serves an important role in the synthesis and excretion of bile acids. A mutant mouse that lacks KLB demonstrates increased synthesis and excretion of bile acids via the elevation of the levels of cytochrome P450 family 7 subfamily A member 1 (CYP7A1) and cytochrome P450 family 8 subfamily B member 1 (CYP8B1) mRNA, which encode the rate-limiting enzymes for the synthesis of bile acids $(8,9)$.

Fibroblast growth factor (FGF)19 acts as a metabolic regulator (10). Endocrine FGF19 functions through the FGF receptor (FGFR) and a co-receptor (either KLA or KLB) (11). KLB may reduce the level of FGF21 through interacting with FGFR4 and FGFR1c $(9,12,13)$. This suggests that KLB is involved in the signal transduction of FGFR4, and serves an important role in the metabolic activity of FGFR4. A previous study demonstrated that the co-expression and activation of KLB in a complex with FGFR4 induced liver cell apoptosis and inhibited hepatoma cell proliferation through activating the signal transduction of extracellular signal-related kinase $1 / 2$, and reducing the signal transduction of protein kinase $B$ (Akt) (14).

KLB was identified to suppress tumor growth in hepatocellular carcinoma via the regulation of the Akt/glycogen synthase kinase $3 \beta$ (GSK-3 $\beta$ )/cyclin D1 signaling pathway (15). In another previous study, it was identified that FGFR4 may suppress the development of breast cancer (16). In addition, KLB shares $41.2 \%$ homology with KLA, and KLA was identified to be a tumor suppressor in human breast cancer (6). Therefore, the present study hypothesized that KLB may be involved in carcinogenesis and may act as a tumor suppressor in breast cancer. The expression and activities of KLB in the mammary glandular and in breast cancer have not yet been elucidated. In the present study, the expression and loss of heterozygosity of KLB in invasive ductal carcinoma was investigated. 


\section{Materials and methods}

Tissue microarray (TMA). An invasive ductal carcinoma TMA (cat. no., OD-CT-RpBre01-006; Outdo Biotech Co., Ltd., Shanghai, China) was performed. The tissue microarray contained tissues from 82 cases, as 328 specimen cores (each with a diameter of $1 \mathrm{~mm}$ and a height of $4 \mu \mathrm{m}$ ), and the integrity of the microarray was $>95 \%$. For all 82 cases, each case included two cores of invasive ductal carcinoma and two cores of paired adjacent non-tumorous breast tissues. All clinical and pathological data of the specimens were provided by Outdo Biotech Co., Ltd., including age, tumor size, whether any axillary lymph node metastasis was present, pathological grade according to the World Health Organization 2012 classification of breast tumors (17) and the staining results of estrogen receptor (ER), progesterone receptor (PR), human epidermal growth factor receptor-2 (HER2) and Ki-67. A total of 48 cases were classified as grade 2 and 34 cases were grade 3. Overall, 37 cases demonstrated lymph node metastasis and 45 cases demonstrated no lymph node metastasis. The age range of patients was 33-88 years (median, 54.5 years) and all patients were female.

Immunohistochemistry. The microarray was washed in $100 \%$ xylene (Beijing Chemical Works, Beijing, China) to remove the paraffin and then rehydrated through serial dilutions of alcohol (100, 90, 80 and 70\%; Beijing Chemical Works) followed by rinsing in water. This was followed by the quenching of endogenous peroxidase activity using a $0.3 \%$ solution of hydrogen peroxide (Beijing Chemical Works) in methanol for $30 \mathrm{~min}$. For antigen retrieval, the section was boiled in $0.01 \mathrm{~mol} / 1$ sodium citrate buffer $(\mathrm{pH}$ 6.0; Beijing Chemical Works) in a microwave oven at $560 \mathrm{~W}$ for $15 \mathrm{~min}$. The section was blocked with $1 \%$ normal goat serum (cat no. ZLI-9021; ZSGB-BIO, Beijing, China) in PBS for $1 \mathrm{~h}$ at room temperature then incubated with anti- $\beta$ klotho antibody (rabbit polyclonal anti-human; cat no. 109454; 1:500 dilution; LifeSpan BioSciences, Inc., Seattle, WA, USA) overnight at $4^{\circ} \mathrm{C}$. The section was laid at room temperature for $30 \mathrm{~min}$ prior to additional analysis the following day. Next, the section was incubated with peroxidase-conjugated goat anti-rabbit IgG secondary antibody (cat no. ZB-2301; 1:250 dilution; ZSGB-BIO) for $30 \mathrm{~min}$. The reaction was visualized using 3,3'-diaminobenzidine (Beijing Chemical Works) under light microscopy (magnification, $x 40$ ) to control optimal dyeing. The section was then counterstained with hematoxylin (Beijing Chemical Works), dehydrated in graded ethanol and xylene, and embedded using permount TM mounting medium (Beijing Chemical Works). The stained TMA was scanned into digital format with the Leica sSCN400 program (Leica Microsystems GmbH, Wetzlar, Germany).

The staining was scored according to the staining intensity and the percentage of the positive tumor cells. The staining intensity was divided into $0,1,2$ and 3 points based on color (no color, faint yellow, brownish yellow and brown, respectively). The percentage of positive tumor cells was divided into four levels $(<5,5-25,26-50,51-75$ and $76-100 \%)$, corresponding to the assignment of $0,1,2,3$ and 4 points, respectively. The product of the scores for staining intensity and the percentage of the positive tumor cells was then used to divide the results into four groups as follows: Negative (-), score 0 ; mild (+), score 1-4; moderate (++), score 5-8; and marked (+++), score 9-12.

Microdissection of breast specimens. The use of all specimens was approved by the Ethics Committee of Capital Medical University (Beijing, China). Invasive ductal carcinoma tissues, paired adjacent non-tumorous breast tissues and lymph nodes were obtained from 42 patients who were diagnosed with primary breast invasive ductal carcinoma. The specimens were collected from between January 2007 to December 2012 at Da Xing Hospital of Capital Medical University, and all patients provided written informed consent. No chemotherapy, radiotherapy or hormone therapy was administered to the patients prior to therapeutic resection. All the tissues were fixed in $10 \%$ formalin (Beijing Chemical Works) at room temperature for $24 \mathrm{~h}$, embedded in paraffin (Beijing Chemical Works) and cut into slices; the paraffin-embedded tissues were cut into 6 sections, each with a diameter of $4 \mu \mathrm{m}$. Next, all sections were stained using hematoxylin and eosin. All cases were reviewed by two pathologists who confirmed the diagnosis of invasive ductal carcinoma and the grading of the tumors according to the 2012 World Health Organization criteria (17). A total of 2 cases were classified as grade 1,16 cases were classified as grade 2 and 24 cases were classified as grade 3 . Overall, 28 cases demonstrated lymph node metastasis and 14 cases demonstrated no lymph node metastasis. Isolating normal epithelial cells and tumor cells with a needle under an inverted microscope, normal epithelial cells and tumor cells were collected and placed into Eppendorf tubes. The same tissue from the same 6 sections were put into the same tube. The genomic DNA was then extracted using QIAamp ${ }^{\circledR}$ DNA mini kit (cat no. 51304; Qiagen GmbH, Hilden, Germany) for loss of heterozygosity $(\mathrm{LOH})$ examination.

$L O H$. A total of 2 microsatellite markers, D4S251 and D4S3040, were selected due to their close proximity to the KLB locus. The 5' polymerase chain reaction (PCR) primers were labeled with 6-carboxyfluorescein. The following PCR primers were used: D4S251 forward, 5'-TATGTATATATG TGTGCGTGCG-3' and reverse, 5'-TATGTATATATGTGT GCGTGCG-3'; and D4S3040 forward, 5'-AGCCTAAGCCTA TCACAATCCAG-3'; and reverse, 5'-CTGATTGGAACCAAG ATGTATATATG-3' (Invitrogen; Thermo Fisher Scientific, Inc., Waltham, MA, USA). PCR was performed as follows: $10 \mathrm{~min}$ at $94^{\circ} \mathrm{C}$, followed by $30 \mathrm{sec}$ at $95^{\circ} \mathrm{C}$, then 40 cycles of $30 \mathrm{sec}$ each at $55^{\circ} \mathrm{C}$ and $30 \mathrm{sec}$ at $72^{\circ} \mathrm{C}$, followed by an extension of $10 \mathrm{~min}$ at $72^{\circ} \mathrm{C}$, in a $20-\mu 1$ reaction mixture containing $0.2 \mu \mathrm{M}$ of each primer, $2.5 \mathrm{ng} / \mu 1$ DNA and $10 \mu 12 \mathrm{X}$ EasyTaq $^{\circledR}$ PCR SuperMix for PAGE (Beijing TransGen Biotech Co., Ltd., Beijing, China). The PCR products were electrophoresed in an ABI Prism 3730 system (Applied Biosystems; Thermo Fisher Scientific, Inc.) according to the manufacturer's protocol and the data were analyzed using the GeneMapper 3.2 software (Applied Biosystems; Thermo Fisher Scientific, Inc.).

Statistics. The statistical analyses were performed with SPSS 19.0 (IBM Corp., Armonk, NY, USA). The correlation between KLB expression and the clinicopathological parameters was evaluated using the $\chi^{2}$ test and Spearman's correlation test. 
$1 \mathrm{~N}$

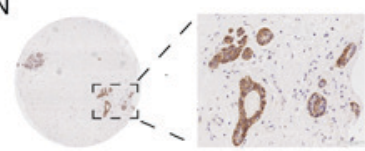

$1 \mathrm{~T}$

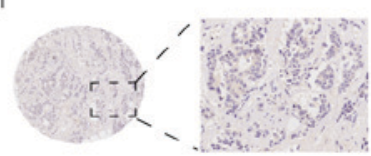

$3 \mathrm{~N}$

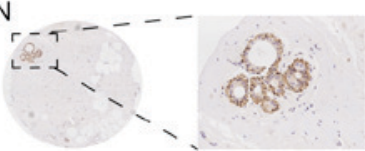

3T

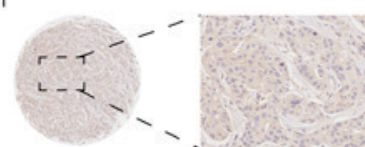

$2 \mathrm{~N}$

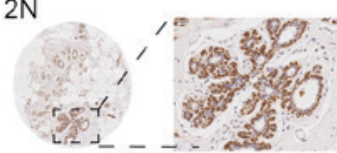

$2 \mathrm{~T}$

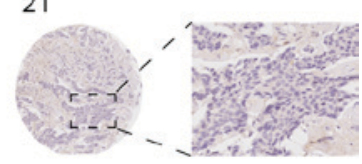

$4 \mathrm{~N}$

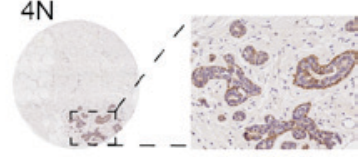

$4 T$

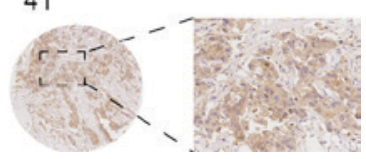

Figure 1. Variable KLB expression levels and locations in invasive ductal tumor and paired adjacent non-tumorous breast tissues. In non-tumorous breast tissues, KLB is primarily expressed in the cell cytoplasm of myoepithelial cells, and the expression is marked. The majority of the tumorous breast tissues exhibited no KLB expression (1T and 2T); certain samples demonstrated mild expression (3T) and moderate expression (4T). Circular images: Original magnification, x40. Rectangular images: Original magnification, $\mathrm{x} 200$. KLB, klotho $\beta$; N, non-tumorous breast tissues; $\mathrm{T}$, tumorous breast tissues.

Two-tailed $\mathrm{P}<0.05$ was considered to indicate a statistically significant difference.

\section{Results}

$K L B$ expression is significantly decreased in invasive ductal carcinoma. In the invasive ductal carcinoma tissues from the TMA, KLB was primarily expressed in the cytoplasm, and in a small number of specimens, KLB was expressed simultaneously in the cell membrane. In general, the expression was identified to be weak, and the rates of negative, mild, moderate and marked expression were 43.90 (36/82), 42.69 (35/82), 10.98 (9/82) and 2.44 (2/82), respectively. In the paired adjacent non-tumorous breast tissues, KLB was primarily expressed in the cell cytoplasm of myoepithelial cells, the expression was marked $(+++)$, and the positive expression rate was $100 \%$ $(82 / 82)$ in the samples. However, mammary glandular epithelial cytoplasm exhibited weak expression (Fig. 1). Overall, KLB expression was significantly decreased in invasive ductal carcinoma tissue compared with the paracancerous tissue $(\mathrm{P}<0.01)$.

Downregulation of KLB is associated with increased lymph node involvement and higher pathological grade. The correlation between KLB expression and a variety of clinical features was analyzed. As summarized in Table I, decreased KLB expression was frequently associated with lymph node involvement $(\mathrm{P}=0.008)$ and pathological grade $(\mathrm{P}=0.022)$. Using correlation analysis, decreased KLB expression was correlated with increased lymph node involvement $(r=-0.234)$

and higher pathological grade $(\mathrm{r}=-0.254)$. However, the expression of KLB was not associated with the age of the patient $(\mathrm{P}=0.551)$ or with the size of the tumor $(\mathrm{P}=0.352)$.

However, KLB expression was not associated with the expression of ER ( $\mathrm{P}=0.894), \mathrm{PR}(\mathrm{P}=0.450)$, HER2 $(\mathrm{P}=0.558)$ or $\mathrm{Ki}-67(\mathrm{P}=0.162)$. The data suggest that KLB expression was also not associated with the secretion of estrogen or progestin, tyrosine kinase activities, or the proliferative index.

$K L B$ locus is subject to $\mathrm{LOH}$ in invasive ductal carcinoma. To examine the mechanisms that regulate the differential expression of KLB in tumors compared with normal tissue, the occurrence of $\mathrm{LOH}$ at this gene locus was examined.

As demonstrated in Fig. 2A, the markers D4S251 and D4S3040 were used to detect $\mathrm{LOH}$ in the patient samples from Da Xing Hospital of Capital Medical University. As summarized in Table II, compared with normal tissue, evidence of LOH was identified in 24/42 tumors (57.14\%); additionally, these two markers were lost in 4/42 tumors $(9.52 \%$; \#9, \#12 and \#15 in Fig. 2B). A total of 28/42 demonstrated lymph node metastasis; $12 / 28$ of these cases (42.86\%) exhibited accompanying $\mathrm{LOH}$ (Table II), and the two aforementioned markers were lost in 2/28 tumors (7.14\%; \#12 and \#15 in Fig. 2B). No association was observed between $\mathrm{LOH}$ and lymph node metastasis or pathological grade $(\mathrm{P}>0.05)$.

\section{Discussion}

KLB is predominantly expressed in the liver, adipose tissue and pancreas (7); however, to the best of our knowledge, its expression in the breast has not been reported. It has been demonstrated that KLB, in a complex with FGFR4, induces liver cell apoptosis and inhibits hepatoma cell proliferation (14), and KLB was suggested to suppress tumor growth in hepatocellular carcinoma via the regulation of the Akt/ GSK-3b/cyclin D1 signaling pathway (15). In another previous study, it was revealed that FGFR4 may suppress the development of breast cancer (16). In addition, KLB shares $41.2 \%$ homology with KLA, and KLA was suggested to be a tumor suppressor in human breast cancer (6). Therefore, the role of KLB in breast cancer was the focus of the present study.

As demonstrated, KLB expression is significantly decreased in invasive ductal carcinoma, and the expression was identified to be weak; the rates of negative and mild expression were 43.9 and $42.69 \%$, respectively. KLB was primarily expressed in the cytoplasm, and in a small number of specimens, it was expressed simultaneously in the cell membrane. In the paracancerous tissue, KLB was primarily expressed in the cell cytoplasm of myoepithelial cells and the expression was marked, however, mammary glandular epithelial cytoplasm exhibited weak expression. This type of KLB expression in invasive ductal carcinoma has not been reported.

In general, tumor cell heterogeneity in the evolutionary process is characterized by differences in morphology and metastasis ability (18). KLB expression in invasive ductal carcinoma tissue is negatively correlated with lymph node metastasis and histological grade; therefore, the weaker the expression of KLB, the greater the level of lymph node metastasis and the higher the histological grade. This also suggests 
Table I. Association between KLB expression and clinicopathological features of patients with invasive ductal carcinoma.

\begin{tabular}{|c|c|c|c|c|c|}
\hline \multirow[b]{2}{*}{ Features } & \multicolumn{4}{|c|}{ Score/klotho $\beta$ expression, $n$} & \multirow[b]{2}{*}{ Overall P-value } \\
\hline & $0 /-$ & $1-4 /+$ & $5-8 /++$ & $9-12 /+++$ & \\
\hline \multicolumn{6}{|l|}{ Age, years } \\
\hline$>55$ & 12 & 19 & 5 & 0 & \multirow[t]{2}{*}{0.551} \\
\hline$\leq 55$ & 23 & 19 & 3 & 1 & \\
\hline \multicolumn{6}{|l|}{ Sizes $(\mathrm{cm})$} \\
\hline$>5$ & 11 & 3 & 1 & 1 & \multirow[t]{3}{*}{0.352} \\
\hline$>2-5$ & 22 & 31 & 8 & 1 & \\
\hline$\leq 2$ & 2 & 2 & 0 & 0 & \\
\hline \multicolumn{6}{|l|}{ Grade } \\
\hline 2 & 16 & 24 & 6 & 2 & \multirow[t]{2}{*}{0.022} \\
\hline 3 & 19 & 12 & 3 & 0 & \\
\hline \multicolumn{6}{|c|}{ Lymph node involvement } \\
\hline Negative & 11 & 25 & 8 & 1 & \multirow[t]{2}{*}{0.008} \\
\hline Positive & 22 & 13 & 1 & 1 & \\
\hline \multicolumn{6}{|c|}{ Estrogen receptor } \\
\hline Negative & 14 & 8 & 6 & 1 & \multirow[t]{3}{*}{0.894} \\
\hline Positive & 13 & 17 & 4 & 0 & \\
\hline Unknown & 10 & 8 & 0 & 1 & \\
\hline \multicolumn{6}{|c|}{ Progesterone receptor } \\
\hline Negative & 15 & 17 & 5 & 1 & \multirow[t]{3}{*}{0.450} \\
\hline Positive & 10 & 11 & 3 & 1 & \\
\hline Unknown & 10 & 9 & 0 & 0 & \\
\hline \multicolumn{6}{|c|}{ Human epidermal growth factor receptor- 2} \\
\hline Negative & 6 & 2 & 2 & 1 & \multirow[t]{3}{*}{0.558} \\
\hline Positive & 21 & 25 & 7 & 0 & \\
\hline Unknown & 9 & 8 & 0 & 1 & \\
\hline \multicolumn{6}{|l|}{$\mathrm{Ki}-67$} \\
\hline 1 & 4 & 13 & 4 & 0 & \multirow[t]{4}{*}{0.162} \\
\hline 2 & 17 & 11 & 2 & 1 & \\
\hline 3 & 5 & 1 & 1 & 0 & \\
\hline Unknown & 10 & 10 & 1 & 2 & \\
\hline
\end{tabular}

+ , mild expression; ++, moderate expression; +++, marked expression.

that KLB may be associated with the heterogeneity, progress and prognosis of invasive ductal carcinoma.

As indicated, in the paracancerous tissue, KLB was primarily expressed in the cell cy toplasm of myoepithelialcells and the expression was marked. Mammary glandular epithelial cytoplasm only exhibited weak expression, therefore, KLB may serve as a biomarker for breast malignancies. It is known that invasive ductal carcinoma originates in the mammary glandular epithelial cells, as there are no myoepithelial cells in tumor tissue. Therefore, the mechanism of this aforementioned expression phenomenon remains to be discussed.

LOH is a common anomaly in the DNA of tumor cells, and it may result in tumor suppressor gene inactivation and then participate in tumor occurrence and development (19). At present, there are no studies concerning the LOH of KLB in invasive ductal carcinoma. In the present study, D4S251 and D4S3040 were selected as KLB markers, and LOH was detected in $57.1 \%$ of cases. This indicates that $\mathrm{LOH}$ may be the mechanism that results in KLB gene inactivation.

There have been a small number of studies investigating KLB function in cancer, which identified that KLA may restrain the progress of breast, lung and kidney cancer $(6,7,20,21)$. Additionally, KLB was suggested to suppress tumor growth in hepatocellular carcinoma (15), and it was revealed that FGFR4 may suppress the development of breast cancer (16), therefore, the present study hypothesized that KLB may inhibit the progress of breast cancer. In our previous unpublished study, the stable breast cancer cell line (MDA-MB-231) in which KLB is overexpressed was successfully cultured. A series of cellular functional experiments, including an MTT viability assay, scratch tests and a colony formation assay, were also 
Table II. List of LOH in tumor and metastasis tissues at D4S251 and D4S3040 markers.

Tissue type

Tumor

Associated metastatic lymph node
Total cases, $\mathrm{n}$
LOH occurrence rate, $\mathrm{n} /$ total n (\%)
LOH occurring at D4S251 and D4S3040 at the same time, $\mathrm{n} /$ total $\mathrm{n}(\%)$

$\mathrm{LOH}$, loss of heterozygosity at the indicated marker.

A

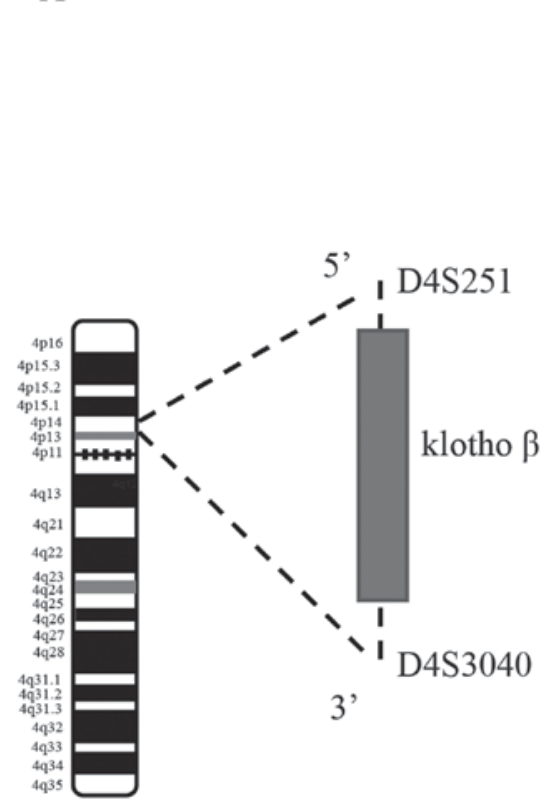

B

$24 / 42(57.14)$

$12 / 28(42.86)$
$4 / 42(9.52)$

$2 / 28(7.14)$

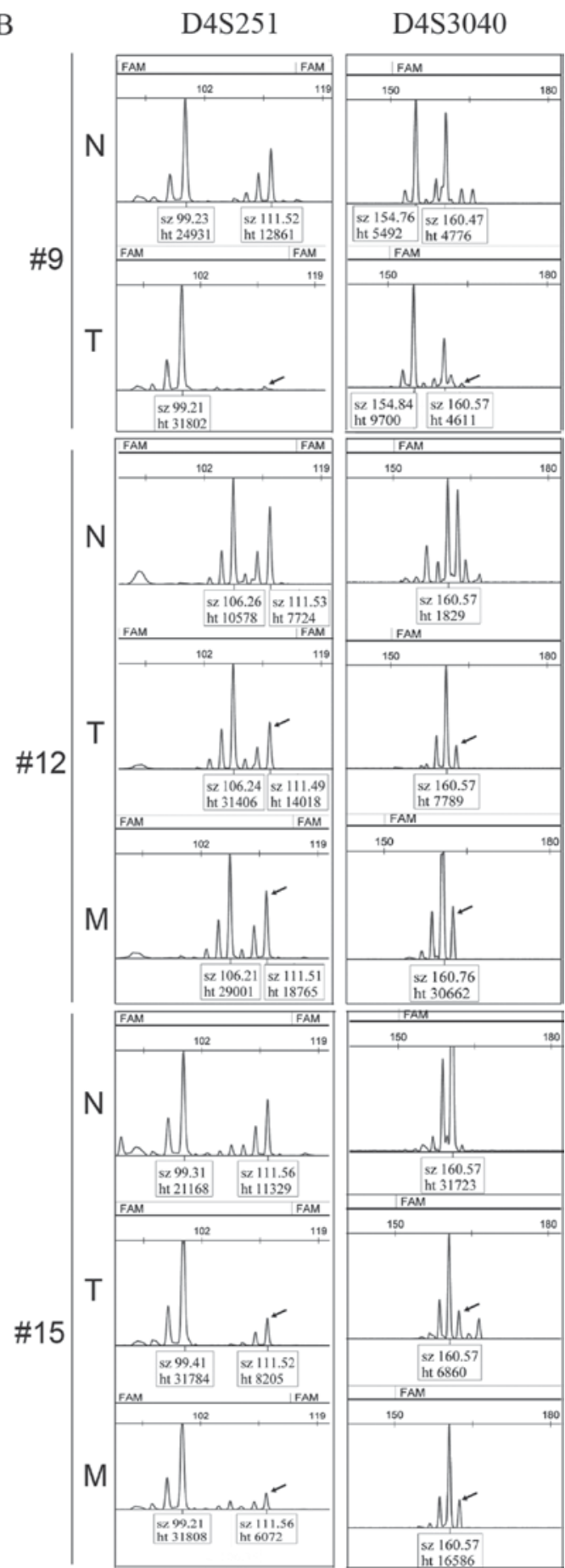

Figure 2. KLB loss of heterozygosity in microdissected invasive ductal carcinoma tissue. (A) KLB gene locus on the short arm of chromosome 4 (4q14), with corresponding markers labeled to the right. (B) Polymerase chain reaction-generated products using the indicated D4S251 and D4S3040 markers are shown. $\mathrm{N}$, normal breast tissue; $\mathrm{T}$, paired microdissected tumor; $\mathrm{M}$, the corresponding metastases. $\mathrm{LOH}$ with loss of two markers is illustrated by tumors (T \#9; $\mathrm{T} \# 12$; M \#12; T \#15; M \#15) compared with normal tissues, as evidenced by loss of peaks (indicated by arrows). 
performed. The experimental group with KLB overexpression was not able to inhibit cell proliferation compared with the control group. It was hypothesized that KLB may serve a role in the suppression of tumor growth in breast cancer, but that this effect would be weak. In a previous study, the differential mRNA expression of sushi, von Willebrand factor type A, EGF and pentraxin domain containing 1, latrophilin 3, KLB, integrin subunit $\alpha 7$, semaphorin $3 \mathrm{G}$, tensin 1 and matrix metalloproteinase 13 genes was examined in breast cancer using reverse transcription-quantitative polymerase chain reaction, and it was demonstrated that the expression of KLB decreased, but that the amplitude was not marked (22). These data are consistent with the results of the present study. Invasive ductal carcinoma originates in the mammary glandular epithelial cells, and the results of the present study indicated that mammary glandular epithelial cytoplasm exhibited weak KLB expression in paracancerous tissue and that KLB expression was weak in invasive ductal carcinoma tissue, which suggests that the hypothesis that KLB suppresses progress in invasive ductal carcinoma requires additional analysis. KLB has been indicated to suppress tumor growth in hepatocellular carcinoma $(15,16)$, however, an additional study demonstrated that KLB expression was frequently upregulated in HCC and that the silencing of KLB expression decreases HCC cell growth (23), therefore, the exact role of KLB in cancer remains unclear.

In summary, KLB expression was decreased in invasive ductal carcinoma in the present study, and this downregulation was correlated with a higher degree of pathology and increased lymph node metastasis. There was a high frequency of $\mathrm{LOH}$ in the KLB gene location, and LOH may be the mechanism that resulted in KLB gene inactivation. KLB may also serve as a particular marker of myoepithelial cells. KLB studies have only investigated its role in HCC, but the role of KLB in the pathogenesis of other malignant diseases should also be investigated.

\section{Acknowledgements}

The authors would like to thank Dr Haye Ding (General Hospital of Beijing Military Region, Beijing, China) for technical assistance. The present study was supported by the National Natural Science Foundation of China (grant no. 81172519).

\section{References}

1. Ferlay J, Soerjomataram I, Ervik M, Dikshit R, Eser S, Mathers C, Rebelo M, Parkin DM, Forman D and Bray F: GLOBOCAN 2012 v1.0, Estimated cancer incidence, mortality and prevalence worldwide: IARC CancerBase No. 11 (Internet). International Agency for Research on Cancer, Lyon, France, 2014. [ http://globocan.iarc.fr/pages/fact_sheets_population.aspx?country=900 ]. Accessed October 9, 2014.

2. National Cancer Institute: Breast Cancer Treatment $\left(\mathrm{PDQ}^{\circledR}\right)$ Patient Version. https://www.cancer.gov/types/breast/patient/ breast-treatment-pdq. Updated May 5, 2017.

3. Kuro-o M, Matsumura Y, Aizawa H, Kawaguchi H, Suga T, Utsugi T, Ohyama Y, Kurabayashi M, Kaname T, Kume E, et al: Mutation of the mouse klotho gene leads to a syndrome resembling ageing. Nature 390: 45-51, 1997.

4. Matsumura Y, Aizawa H, Shiraki-Iida T, Nagai R, Kuro-o M and Nabeshima Y: Identification of the human Klotho gene and its two transcripts encoding membrane and secreted Klotho protein. Biochem Biophys Res Commun 242: 626-630, 1998.
5. Shiraki-Iida T, Aizawa H, Matsumura Y, Sekine S, Iida A, Anazawa H, Nagai R, Kuro-o M and Nabeshima Y: Structure of the mouse klotho gene and its two transcripts encoding membrane and secreted protein. FEBS Lett 424: 6-10, 1998.

6. Wolf I, Levanon-Cohen S, Bose S, Ligumsky H, Sredni B, Kanety H, Kuro-o M, Karlan B, Kaufman B, Koeffler HP and Rubinek T: Klotho: A tumor suppressor and a modulator of the IGF-1 and FGF pathways in human breast cancer. Oncogene 27: 7094-7105, 2008

7. Ito S, Kinoshita S, Shiraishi N, Nakagawa S, Sekine S, Fujimori T and Nabeshima YI: Molecular cloning and expression analyses of mouse betaklotho, which encodes a novel Klotho family protein. Mech Dev 98: 115-119, 2000.

8. Ito S, Fujimori T, Furuya A, Satoh J, Nabeshima Y and Nabeshima Y: Impaired negative feedback suppression of bile acid synthesis in mice lacking betaKlotho. J Clin Invest 115: 2202-2208, 2005.

9. Ogawa Y, Kurosu H, Yamamoto M, Nandi A, Rosenblatt KP, Goetz R, Eliseenkova AV, Mohammadi M and Kuro-o M: BetaKlotho is required for metabolic activity of fibroblast growth factor 21. Proc Natl Acad Sci USA 104: 7432-7437, 2007.

10. Fukumoto $\mathrm{S}$ : Actions and mode of actions of FGF19 subfamily members. Endocr J 55: 23-31, 2008.

11. Tomiyama K, Maeda R, Urakawa I, Yamazaki Y, Tanaka T, Ito S, Nabeshima Y, Tomita T, Odori S, Hosoda K, et al: Relevant use of Klotho in FGF19 subfamily signaling system in vivo. Proc Natl Acad Sci USA 107: 1666-1671, 2010.

12. Kurosu H, Choi M, Ogawa Y, Dickson AS, Goetz R, Eliseenkova AV, Mohammadi M, Rosenblatt KP, Kliewer SA and Kuro-o M: Tissue-specific expression of betaKlotho and fibroblast growth factor (FGF) receptor isoforms determines metabolic activity of FGF19 and FGF21. J Biol Chem 282: 26687-26695, 2007.

13. Lin BC, Wang M, Blackmore C and Desnoyers LR: Liver-specific activities of FGF19 require Klotho beta. J Biol Chem 282: 27277-27284, 2007.

14. Luo Y, Yang C, Lu W, Xie R, Jin C, Huang P, Wang F and McKeehan WL: Metabolic regulator betaKlotho interacts with fibroblast growth factor receptor 4 (FGFR4) to induce apoptosis and inhibit tumor cell proliferation. J Biol Chem 285: 30069-30078, 2010.

15. Ye X, Guo Y, Zhang Q, Chen W, Hua X, Liu W, Yang Y and Chen G: $\beta$ Klotho suppresses tumor growth in hepatocellular carcinoma by regulating Akt/GSK-3 $\beta /$ Cyclin D1 signaling pathway. PLoS One 8: e55615, 2013.

16. Zhu X, Zheng L, Asa SL and Ezzat S: Loss of Heterozygosity and DNA methylation affect germline fibroblast growth factor receptor 4 polymorphism to direct allelic selection in breast cancer. Am J Pathol 177: 2860-2869, 2010.

17. Lakhani Sunil R; International Agency for Research on Cancer; World Health Organization, Lyon :International Agency for Research on Cancer, 2012.

18. Marusyk A,Almendro V and Polyak K: Intra-tumour heterogeneity: A looking glass for cancer? Nat Rev Cancer 12: 323-334, 2012.

19. Yang CY, Lu RH, Lin CH, Jen CH, Tung CY, Yang SH, Lin JK, Jiang JK and Lin CH: Single nucleotide polymorphisms associated with colorectal cancer susceptibility and loss of heterozygosity in a taiwanese population. PLoS One 9: e100060, 2014.

20. Chen B, Wang X, Zhao W and Wu J: Klotho inhibits growth and promotes apoptosis in human lung cancer cell line A549. J Exp Clin Cancer Res 29: 99, 2010.

21. Doi S, Zou Y, Togao O, Pastor JV, John GB, Wang L, Shiizaki K, Gotschall R, Schiavi S, Yorioka N, et al: Klotho inhibits transforming growth factor-betal (TGF-beta1) signaling and suppresses renal fibrosis and cancer metastasis in mice. J Biol Chem 286: 8655-8665, 2011.

22. Kotepui M, Thawornkuno C, Chavalitshewinkoon-Petmitr P, Punyarit P and Petmitr S: Quantitative real-time RT-PCR of ITGA7, SVEP1, TNS1, LPHN3, SEMA3G, KLB and MMP13 mRNA expression in breast cancer. Asian Pac J Cancer Prev 13: 5879-5882, 2012.

23. Poh W, Wong W, Ong H, Aung MO, Lim SG, Chua BT and Ho HK: Klotho-beta overexpression as a novel target for suppressing proliferation and fibroblast growth receptor-4 signaling in hepatocellular carcinoma. Mol Cancer 11: 14, 2012. 